\title{
Home Improvement: Evaluating Secular Changes in NLSY HOME- Cognitive Stimulation and Emotional Support Scores
}

\author{
Patrick O'Keefe $\mathbb{i}^{1} \cdot$ Joseph Lee Rodgers ${ }^{2}$
}

Received: 18 March 2021 / Accepted: 20 October 2021 / Published online: 3 November 2021

(c) The Author(s), under exclusive licence to Springer Science+Business Media, LLC, part of Springer Nature 2021

\begin{abstract}
This study evaluated changes over time in the quality of children's home environment, using the Home Observation Measurement of the Environment (HOME). Longitudinal increases in HOME scores were predicted by both theory and past empirical results. Analysis of the National Longitudinal Survey of Youth Children data $(\mathrm{N}=5715$, aged 0-14) suggested that HOME scores have been increasing, and that the increase is a family-level phenomenon. The data were a sample of children born to mothers who were approximately representative of the United States in 1979. An increase in HOME scores occurred primarily for the three age categories younger than ten. Effect sizes were of approximately the same magnitude as the Flynn effect for intelligence. These results have implications for policy and future research regarding the home environment.
\end{abstract}

Keywords $\mathrm{HOME} \cdot$ Children's home environment $\cdot$ longitudinal changes $\cdot$ cultural ratchet $\cdot$ Flynn effect

\section{Highlights}

- This study examined changes in the home environment using a large representative U.S. sample, trends were found spanning 30 years

- The trends discovered were consistent with other secular trends associated with the Flynn effect

- Maternal age at first birth was a particularly important predictor of children's home environment

We begin with a simple question: Has the quality of children's home environment improved over time? We find very little earlier treatment of this question, and suggest that answering it is valuable to researchers and policy makers. For researchers, should it be found that the home environment has improved substantially over time, the logical next step is to pursue the cause of this improvement. Policy makers should likewise be interested in funding and implementing effective programs to further improve, or maintain improvements, in the home environment

Patrick O'Keefe

okeefep@ohsu.edu

1 Department of Neurology, Oregon Health \& Science University, 3181 SW Sam Jackson Park Road, L226, Portland, Oregon 972393098, USA

2 Vanderbilt University-Department of Psychology and Human Development, Peabody College \#552, 230 Appleton Place, Nashville, TN 37203-5721, USA of children. In pursuing this question, we begin to address some potential causes of such an increase, although we do not pin down a single cause (further theoretical development is left for future research).

For children, one of the most important developmental contexts is the home environment. The home environment comprises both material resources (e.g., books, television, and toys) and parental factors (e.g., supervision, parental warmth, parental discipline). The concept is necessarily broad, as the environment encompasses the entire context in which a child exists, and we are only limiting ourselves to the portion of a child's environment that exists in the home. In the present study we investigate whether the home environment, broadly defined, has experienced secular changes, using a sample of children living in the United States. We motivate our hypothesis proposing secular changes (long term, population level changes) on the basis of previously observed changes that can reasonably be linked to the home environment. We test this hypothesis using a large, representative, longitudinal U.S. survey that 
includes a commonly used measure of the home environment. Though broad longitudinal changes in the home environment have not been studied earlier, we have identified a number of prior studies on parent and childhood outcomes that are linked to the home environment, such as mathematical skills (Susperreguy et al., 2020) and problem behaviors (Rodgers et al., 1994), suggesting that the home environment plays an important role in development. Furthermore, policy changes such as improved access to Head Start programs, home visitation programs, or tax credits, might reasonably be expected to have an impact on the home environment, although past research has shown mixed results (e.g., Hamad \& Rehkopf, 2016; Jones Harden et al., 2012; Kendrick et al., 2000).

In the current study, we evaluate empirical evidence to assess whether children's home environments in the U.S. have been improving between the mid 1980's and the mid 2010's (the period of observation covered by our data). There has been a push in behavioral science research to address long-term trends in other outcomes. Some of these changes have implications-in an indirect manner-for the current study. Such sustained changes are commonly referred to in the behavioral science literature as secular changes (e.g., Mingroni, 2007). As an example of some secular trends, over the past century researchers have identified a sustained increase in intelligence (usually referred to as the "Flynn effect"; see Flynn, 1987), increases in height (Bielecki et al, 2012), even faster gains in weight (Fredricks et al., 2000; Loesch et al., 2000), and increases in rates of several mental health issues. In athletics, as time moves forward world records in track events continue to drop, tennis serves are hit harder, and footballs are kicked further. At the national level, technological improvement and Gross Domestic Product (GDP) have increased substantially. The most carefully studied of these secular changes involves intelligence, where changes over time have been documented as early as we can measure intelligence (e.g., Lynn, 2009). The types of changes documented in this past research would be anomalous if nothing was changing in the home environment of children, which helps to motivate our hypothesis.

The home environment is the primary developmental context of children, particularly young children, and empirical work demonstrates the impact of the home environment on child development. For example, Strauss and Knight (1999) showed that the home environment was a consistent predictor of children's obesity status six years after initial observation. The home environment remained a significant predictor of obesity even after controlling for initial BMI, and a variety of maternal factors including maternal BMI, occupation, and education. The home environment has been found to partially mediate the effect of parental education on children's academic outcomes
(Davis-Kean, 2005). The home environment also partially mediates the effect of socio-economic status on children's inhibitory control and working memory (Sarsour et al., 2011). In a study of group differences, Brooks-Gunn et al. (1996) found that including a measure of the home environment reduced the IQ gap between Black and White children by a significant amount. Research by Bono et al., (2016) has found significant impacts for maternal time investments on children's cognitive outcomes. Parental time investments form a significant part of the home environment of children, and the measure we rely upon here includes a number of questions regarding parental behaviors. Adding complexity to the intuitive but simple "environment leads to outcome" model, Hadd and Rodgers (2016) found that children's intelligence contributes to constructing their own home environment. This finding suggests that the environment consists not only of the material and behaviors external to the child (e.g., parental warmth, parental income), but is at a more nuanced level composed of the interplay of material resources, parental (and other caregiver) behaviors, and the child's own behavior (see Scarr \& McCarthy, 1983, for an earlier theoretical statement of this principle). Similarly, recent research suggests that associations between the home environment and child outcomes may reflect genetic effects of parents that shape both the child outcomes (via shared genetic pathways) and the home environment itself (via parental choices influenced by their own genetic predispositions; e.g., Puglisi et al., 2017; van Bergen et al., 2017). In summary, the home environment is associated, to a meaningful degree, with a number of important child outcomes, and can be influenced even by those very outcomes through potentially complex feedback loops.

Measuring the home environment entails measuring a number of different, and sometimes disparate, features of that environment. Virtually any meaningful human environment involves the interaction of both human behaviors and material things. The measure we use, the HOME Short Form, a shortened version of the Home Observation for Measurement of the Environment (HOME; Caldwell \& Bradley 1984), measures aspects of the home environment as wide ranging as the count of books, the number of age appropriate toys, trips to the museum, television viewing habits, and how the parent interacts with their child during the survey interview. The inclusion of such wide ranging evaluations in a single measure allows for comprehensive evaluation of the environment; however, it does create measurement issues from a psychometric perspective.

From a measurement perspective any measure of the home environment is unlikely to be unidimensional, which raises the possibility that one dimension of the home environment may have increases while another has declines, or that the strength of increase in one is not matched by the 
strength of increase in other dimensions. For example, the HOME-SF has both a cognitive stimulation and an emotional support subscale that are created from the overall form. The general implication is that, should we find secular changes, such changes may not reflect a uniform change across different dimensions of the home environment. Our study represents a preliminary and partially exploratory investigation, and thus such psychometric challenges pose fewer issue than if this were a strongly confirmatory study. Obviously, our findings will not be the final word on the topic, as the home environment is a rich, multi-faceted, context and each facet deserves careful study.

\section{Expectation of Secular Changes: Generating Working Hypotheses}

We now explore in more depth why we might expect changes in the home environment. We begin by outlining very general theories that would predict change. We then move on to theories and empirical results that predict the direction and strength of change we may expect in the home environment.

The cultural ratchet hypothesis (e.g., Tennie et al., 2009; Tomasello et al., 1993) is a broad theory for why human society is generally much more sophisticated than even our closest animal relatives. Basically, the theory rests on the fact that humans transmit knowledge and innovations to each other. If one person develops a beneficial, trainable, behavior, that behavior can be transmitted to other humans. If a behavior proves useful it can be preserved and transmitted from one generation to the next. This process of innovation, dissemination, and preservation relies on traits that, although perhaps not completely unique to humans, are developed to their highest extent in humans. First, innovation is necessary. For a new behavior to come about someone must be the first to think of (and perform) that behavior. After the innovation occurs it must be disseminated to others. Previously this would have required one human showing another (in person) how to perform the new behavior. Today, we are able to transmit written or verbal instructions and visual demonstrations for new behaviors throughout the world nearly instantly. Finally, for a behavior to persist it must be reproduced faithfully and with fidelity. Previously this process would have relied on human ability to memorize the behavior, but today, with the various means of recording information (e.g., written word, video, audio etc.), reproducing behavior is simpler as it simply can be copied from existing records (instead of from memory). To the extent that a behavior is advantageous or otherwise desirable this process serves to move society towards that behavior. This mechanism of innovation, transmission, and preservation serves to create the "cultural ratchet". Useful behaviors advance society, and society prevents regress by the faithful transmission and preservation of those behaviors.

The cultural ratchet hypothesis is quite general, but from this basic idea (innovation, transmission, replication) we would reasonably expect positive changes in the family environment. For the home environment we would expect parents to desire good outcomes for their children, and to eagerly and carefully implement innovation that supports such outcomes. So long as improvements can be made to the home environment, and so long as the home environment fosters desirable outcomes for children, the cultural ratchet hypothesis would predict a generally upward trend in the home environment. The cultural ratchet hypothesis provides a broad grounding and general motivation for our hypothesis, and even a directional orientation. Other literature documents more specific trends that may further inform our research.

Before continuing, it is important to note that our measure of the home environment includes a subscale comprised of items related to cognitive development (cognitive stimulation), and a subscale comprised of items related to emotional outcomes (emotional support). Our hypothesis, to this point, is not constrained by these measures. To the extent that a given outcome is related to the home environment (either as a cause of the home environment, or as an outcome of it) similar hypotheses could be formed. So, for example, if athletic ability were of interest to a researcher, and they believed that the home environment caused (or was related to) athletic ability, then to the extent that athletic ability had improved we would hypothesize associated improvements in the home environment. As outcomes become more specific the aspects of the home environment that would be expected to change might narrow. However, at present we are examining two broad categories, cognitive stimulation and emotional support, and so the elements of the home environment that we expect to change are also quite broad.

With regards to cognitive stimulation, we do not need to look far for trends that might implicate changes in the home environment. The Flynn effect is the well-documented phenomenon that measured IQ has increased, substantially, over (at least) the last 100 years (e.g., Flynn, 1984, 1987, 2018; Lynn, 1982, 2013). A number of plausible reasons for this increase have been proposed, however no one explanatory theory has received unanimous support (e.g., Rodgers, 1998, 2015). Despite the lack of consensus regarding the precise cause of the Flynn effect, several theories would suggest that we might expect improvements in the home environment, at least as it relates to cognitive development. For example, Lynn (2009) suggested that improved neonatal and early post-natal nutrition might cause the Flynn effect. Dickens and Flynn, (2001) suggest that we have improved 
the ability for individuals, especially children, to find and fill niches for which they are uniquely suited. To the extent that the home environment has previously fallen short of the needs of children, this theory would predict both an improved home environment generally, and one that matches an individual child's needs more specifically. This finding also matches findings of Hadd and Rodgers (2016) that a child's home environment changes in response to characteristics of the child. A cross-generational feature of the Flynn effect suggests that we may expect parents to provide progressively better environments as we expect more intelligent parents to provide better environments for their children. Finally, because cognitive ability has both genetic and environmental main effects, and also gene $\mathrm{X}$ environment interactions (Turkheimer et al., 2003) an increase in societal cognitive ability would suggest the possibility of a changing developmental context. In general, the Flynn effect and research on its causes are suggestive that the home environment, at least as it relates to cognitive stimulation, has likely seen improvements over the past few decades.

The Flynn effect serves as a touchstone for comparison throughout this paper. There are a few reasons for this. First, the Flynn effect is relatively easy to understand (IQ scores are increasing over time), and as our theory is of a substantially similar nature (the home environment has been improving over time) it serves as a convenient comparison. Second, the Flynn effect is thoroughly documented and evaluated, and provides a convenient yardstick by which to measure the magnitude of a secular change, with a $0.02 \mathrm{SD}$ change per year being called "massive" in the literature (e.g., Flynn, 1984). Third, the existence of the Flynn effect provides motivating evidence for our hypotheses regarding the home environment as it relates to cognitive outcomes.

Emotional support is also an interesting and accessible feature of the home environment. On this front we have less literature from which to draw to posit improvements in the home environment. However, we note that many of the reasons we have given for general improvements in the home environment, and the home environment as it applies to cognition and cognitive stimulation, would also predict positive changes in the home environment as it applies to emotional support and related outcomes. The cultural ratchet hypothesis is not limited to cognitive outcomes; it applies to behavior generally. Several of the Flynn effect theories could easily be altered to predict better emotional outcomes as well. For example, parents would likely care for their children's emotional well-being as much as their child's cognitive competence, which could lead parents actively working to help increase and improve their children's emotional support. Better nutrition could contribute to emotional outcomes as effectively as it does to cognitive outcomes. In practice, improvements in emotional support might be observed in, for example, shifts in parental attitudes away from corporal punishment and other harsh parenting practices, even among groups historically more favorable to such practices (e.g., Hoffman et al., 2017). Other changes that might have impacted children's emotional support include changes in both mother's and father's time investment in their children (e.g., Sayer et al., 2004). These shifts, and others, may lead to improvements in children's emotional support and related outcomes. However, there are also reasons to believe that, perhaps, positive increases are not the whole story. Diagnoses of anxiety and depression have risen substantially in recent decades (e.g., Collishaw, 2015; McLaughlin et al., 2007). The prevalence of autism spectrum disorders also appears to be on the rise (e.g., Blumberg et al., 2013; Croen et al., 2002). These patterns are not the outcomes that would be expected from an environment that is improving with regards to emotional support, which suggests to us that we should not, a priori, necessarily expect the same sorts of gains in the emotional support subscale of the HOME-SF as we expect in the cognitive stimulation subscale. In fact, given the increasing prevalence of negative emotional outcomes (e.g., higher rates of depression), we may even expect negative trends. If the scores on the HOME-SF emotional support subscale are found to be increasing, it is possible that items measured by the HOME-SF emotional support subscale do not impact children's emotional development, or that higher scores do not necessarily result in better outcomes.

\section{The Present Study}

The present study considers whether the home environment, broadly defined, has experienced secular changes-a new research question that, we believe, has not previously been addressed. We do so by examining a longitudinal dataset that spans approximately 30 years. We do not expect large year over year changes - for example, the magnitude of the Flynn effect is approximately $0.02 \mathrm{SD}$ (0.3 IQ points) per year (e.g., Pietschnig \& Voracek, 2015)—but we do predict sustained changes. To the extent that the home environment is related to cognitive stimulation we would predict a generally positive increase, that is, our hypothesis is directional. To the extent that the home environment is related to emotional support we are more agnostic about predicting change, or its direction. The cultural ratchet would predict that improvements should generally occur in society, however, measurable outcomes related to depression and mental health provide challenges suggesting that children and teens may be experiencing an environment that is declining compared to previous ones (although the relevant environment in this case may not be the home environment).

Further refining our hypothesis, research by O'Keefe and Rodgers (2017), using the same source of data as used here, found that the Flynn effect may be a largely family-based 
effect, and perhaps linked to mother's age at first birth. Similarly, Fulco et al. (2019) found that mother's age at first birth was linked to a measure of the home environment, also in this source of data. On the basis of these two studies we would predict that, if we find a longitudinal increase it is likely to be linked to mother's age at first birth. In addition to time, we also account for parental income, maternal education and maternal cognitive ability, in part to control for potential selection effects, and also because the effects of these covariates are interesting in their own right. However, these covariates also encompass much of what is assumed to be potentially beneficial for mothers who delay childbearing, so why include maternal age at first birth? The first reason is methodological, and is explained in greater detail in the methods section, but succinctly maternal age is naturally included in our model as part of a set of timerelated variables, and excluding it would lead to an incomplete and somewhat incoherent set of these variables. The second reason is practical: in previous research (e.g., O'Keefe and Rodgers, 2017) maternal age at first birth still had substantial explanatory power even after accounting for other maternal effects (e.g., cognitive ability and income). We return to this phenomenon in our discussion.

We confine ourselves to linear patterns of change in our analyses. It is certainly possible, indeed plausible, that change occurs non-linearly. For example, Pietschnig and Voracek (2015) found non-linear trends in the Flynn effect. It is entirely possible that similar non-linearities exist for changes in the home environment as well. However, as our hypotheses posit monotonic changes, linear tests would provide at least preliminary evaluation of our hypotheses. Additional non-linear effects, while interesting in their own right and more nuanced, would not serve to address our specific research questions.

\section{Methods}

\section{Participants}

Our samples come from the National Longitudinal Survey of Youth (NLSY), including the NLSY-children (NLSYC) and their NLSY mothers (females from the NLSY79).
The NLSY79 surveyed men and women ages 14-22, starting in 1979; the longitudinal collection of this dataset is still ongoing and available online through 2018. The original NLSY79 sample was based on a household probability sample. Additional oversamples of military, Hispanic, Black, and poor White respondents were also included in addition to the household probability sample. The NLSY79 respondents have been followed on an annual basis until 1994, and a biennial basis since. In 1986, the biological children of the women from this sample were surveyed, and all biological children of the NLSY79 females have been followed on a biennial basis since then.

In the NLSYC there are over 13,000 children, representing thousands of families, spread over more than two decades of observation. Retention is generally quite good, with $63 \%$ of children completing at least six (of a maximum eight possible) interviews (Bureau of Labor Statistics n.d.). It is worth noting that because of the study design, not all children would have been eligible to complete the maximum number of interviews. For the purposes of our analysis we restricted ourselves to children of the original household probability sample (which was approximately half of the NLSY79, excluding the oversamples). Because all children were biological children of the NLSY79 women, none were adopted or foster children. This approach provides a sample that is representative (up to attrition and design adjustments) of the children of women who were living in the U.S. and age 14-22 in 1979. This selection criterion approximately halves the available data, but reduces concerns of selection bias associated with the oversamples. Because of the design of the measurement instruments available to us, subjects were split into four age groups. This was necessary because the HOME measure changes as children age to account for the changing relevance of various environmental factors. The age groups are $0-2,3-5,6-9$, and 10-14. In the NLSYC data, at younger ages children's race data is assigned to be the same as their mother's, who self-reports race. The three categories were Hispanic, Black, and non-Black-non-Hispanic, and responses were limited to a single selection. For the present study we use the mother's reported race as a proxy for child's race. The race and sex demographics for each age group are presented in Table 1; values are approximate and
Table 1 Maternal race and child sex sample statistics by age group

\begin{tabular}{lllllll}
\hline Age Group & Hispanic & Black & $\begin{array}{l}\text { Neither Hispanic } \\
\text { Nor Black }\end{array}$ & Male & Female & $\begin{array}{l}\text { Spouse or partner of mother } \\
\text { present }^{\mathrm{a}}\end{array}$ \\
\hline $0-2$ & 0.08 & 0.12 & 0.81 & 0.51 & 0.49 & 0.87 \\
$3-5$ & 0.08 & 0.12 & 0.79 & 0.51 & 0.49 & 0.82 \\
$6-9$ & 0.08 & 0.14 & 0.78 & 0.52 & 0.48 & 0.81 \\
$10-14$ & 0.08 & 0.14 & 0.77 & 0.52 & 0.48 & 0.78 \\
\hline
\end{tabular}

${ }^{a}$ This measures if the spouse or partner of the mother was present at any wave of data collection during the span of years the child was in a given age group. The spouse or partner may not be the father of the child 
may not add to $100 \%$ due to rounding. (We note that Ang, Wanstrom, and Rodgers, 2010, found no race or gender differences in NLSYC Flynn effect patterns.) For all age groups the mother's spouse or partner was present in the household for at least one wave of observation for approximately $80-85 \%$ of children. The Vanderbilt University institutional review board (IRB) ruled this research exempt, because it is based on public data that are deidentified and archival.

\section{Measurement}

The Home Observation Measurement of the Environment (HOME; Caldwell \& Bradley 1984) questionnaire provides a general measure of the home environment that has been widely used in behavioral science research settings (e.g., Elardo \& Bradley, 1981). Although initially developed for young children, later development resulted in measures for older aged children (e.g., Bradley et al., 1988). The NLSYC uses a shortened version of the HOME, the HOME-SF. The HOME-SF has been extensively studied and used, both as an independent and/or dependent variable, in hundreds of research studies. Mott (2004), provides an overview of this research including information on the reliability of the measure in the NLSYC specifically and how it has been used in previous research. Because the research reviewed by Mott relies on the NLSYC the results can be considered to come from a subset of the data we analyze subsequently. Due to the longitudinal nature of the NLSYC our data include additional observations that could not have been included by the research reviewed by Mott (2004), but given the extensive overlap between our sample and the sample accessible prior to 2004 we believe that the findings in Mott are directly applicable to our current work. According to Mott (2004), reliability of the measure is relatively low for the very youngest age group (approximately $0.50-0.60$ ), but is higher for the older age groups (approximately 0.70-80). Mott suggests, and we agree, that lower reliability for the younger children is likely due to both a smaller question set and lower completion rates for the youngest age group. Included in the NLSYC are two HOME subscales, a cognitive stimulation subscale and an emotional support subscale, each of which is measured longitudinally from 1986-2014. Mott (2004), indicated that the cognitive stimulation subscale is generally more reliable than the emotional support subscale in the NSLYC.

The HOME-SF question set changes as children age. The items are a mix of likert items, binary (yes/no) responses, and counts of behaviors. The responses are provided by both home observers (objective raters) as well as parents and, for older ages, the NLSYC children themselves, depending on the item. An example item from the cognitive stimulation subscale is "About how many children's books does [the] child have?" For the emotional support subscale an example item is "How often does [the] child eat a meal with both you and his/her father/step/ father-figure?" Items with more than two valid responses are collapsed into a binary response and the subsequent 0 / 1 scores are summed. The NLSYC subsequently multiplies all scores by 10 . Higher scores indicate a better home environment, and a 10-point increment indicates a single unit improvement in the NLSYC HOME data. There is a separate set of questions for children ages 0-2, 3-5, 6-9, and 10-14. As the number and set of questions change across ages there exist different maximum scores across ages. The administration of the HOME was changed in 2006 such that children younger than 4 were given the interviewer-administered assessments, which made it necessary to drop those children from 2006 forward for this analysis. Due to the pattern of births in the NLSYC this adjustment does not substantially reduce the sample size. Because of the varying nature of the measure across ages a separate analysis was conducted for each age group and for each of the two subscales.

\section{Missing Data}

In each set of observations there were missing data. We used multiple imputation to address this. Sample sizes enumerated by model level and age group can be found in Table 2, the minimum sample size is greater than 2,000, whereas the largest sample size is over 12,000. Separate analyses of the two subscales were conducted, to separately identify the potential for secular increases in one or the other (or both or neither).

The percentage of cases with complete data are shown in Table 3. We used multiple imputation via the "mice" package in R (Buuren, \& Groothuis-Oudshoorn, 2010) and the "miceadds" package (Robitzsch, \& Grund, 2020) to account for the missing data. The mice package allows for sophisticated procedures to impute missing data, including when data are multilevel. For each set of observations we created 50 multiply imputed datasets. The results from each dataset were pooled using available functions from the mice package. For all cases the most frequently missing data were the HOME outcome, followed by parental income, maternal cognitive ability, and maternal education.

Table 2 Sample sizes at each level for each age group

\begin{tabular}{llll}
\hline Age Group & Observations & Children & Families \\
\hline $0-2$ & 5608 & 4045 & 2089 \\
$3-5$ & 6787 & 4787 & 2285 \\
$6-9$ & 10,001 & 5306 & 2369 \\
$10-14$ & 12,339 & 5246 & 2317 \\
\hline
\end{tabular}


Table 3 Complete case percentage for each measure at each age

\begin{tabular}{lll}
\hline & Emotional Support & Cognitive Stimulation \\
\hline $0-2$ & 0.63 & 0.69 \\
$3-5$ & 0.69 & 0.69 \\
$6-9$ & 0.67 & 0.69 \\
$10-14$ & 0.55 & 0.6 \\
\hline
\end{tabular}

Attrition was a concern. To evaluate the effect of attrition cases where observations were intermittent (i.e., cases where a given child was not observed every two years as planned) or where children appeared to have dropped out (cases where children were younger than 13 years of age at their final observation, but the final observation was prior to the final year of collected data) we examined group differences on income, maternal AFQT score, maternal education, and HOME subscale scores using $t$-tests. Although there are instances where a family appears multiple times in the comparison (potentially inflating the Type I error rate), our concern is with the magnitude of the differences rather than merely statistical significance. Group differences on all three variables had Cohen's $d<0.1$, implying small effects. Group differences were only statistically significant for education $(p<0.05)$.

\section{Statistical Modeling}

Models were fit with the "Ime4" package in R (Bates et al., 2014). The lme 4 package provides a suite of functions for the fitting of multilevel models. In the present case observations are nested within children, who are themselves nested within families. This nesting structure requires the use of appropriate modeling techniques to properly estimate both effects and standard errors to account for correlated error terms caused by the nesting structure.

Models were run separately by age category with the selected HOME scale predicted by year of testing and a set of control variables described below. After this initial modeling we fit a second set of multilevel models using a variable centering method developed and described in O'Keefe and Rodgers (2017). This methodological procedure allows orthogonal between-level and nonorthogonal within-level decomposition of variables. This structure allows separation of effects into descriptive processes across multiple levels. Succinctly, this variable centering method pinpoints where in the multilevel structure of the data the time-related change is taking place. In this analysis the year of observation was partitioned according to the method in O'Keefe and Rodgers. The same model was used across outcomes and age groups. Equation 1 shows the model used. The "Score" is the HOME outcome being modeled (e.g., HOME subscales).
The independent variables (income, education, AFQT, etc.) are described next.

Equation 1: Model of HOME outcome.

$$
\begin{aligned}
\text { Score }_{i j k}= & \beta_{0}+\beta_{1} \times \text { Income }_{i k}+\beta_{2} \times \text { Education }_{i k} \\
& +\beta_{3} \times \text { AFQT }_{k}+\beta_{4} \times \text { Mother' }_{\text {sAgeFirstBirth }} \\
& +\beta_{5} \times \text { Mother' }^{\prime} \text { BirthYear }_{k}+\beta_{6} \times \text { FamilyMeanAge }_{k} \\
& +\beta_{7} \times \text { FamilyMeanSiblingDifference }_{k} \\
& +\beta_{8} \times \text { ChildMeanAge }_{j k}+\beta_{9} \times \text { SiblingDifference }_{j k} \\
& +\beta_{10} \times \text { ChildAge }_{i j k}+e_{i j k}
\end{aligned}
$$

In addition to the HOME as an outcome variable and the decomposition of time as independent/explanatory variables, three control variables were included in every model to adjust for the time-based confound that older women on average have higher cognitive ability, income, and education. This adjustment is a routine and important adjustment for NLSY research (see Rodgers \& Wanstrom, 2007, for an expanded rationale). A measure of cognitive ability, the Armed Forces Qualifying Test (AFQT; Ree et al., 1982) was administered to mothers at the beginning of the study in 1980 when they were between 15 and 23 years of age. Because the AFQT was designed for adults, age norms were created by the NLS staff for each age group in the NLSY79, and these normed scores were used in our models. Maternal education was measured as the highest grade completed by a given survey period, and was included as a control variable. The last variable included as a control was total net family income, which adds the income from adult members of the household for the previous year, adjusted for inflation using the consumer price index. These three variables directly account for the selection bias previously mentioned.

The double decomposition model used is presented and outlined (including an empirical analysis) in greater detail in O'Keefe and Rodgers (2017), and thus it is only briefly summarized here. The variable "year of observation" can be broken down (i.e., algebraically decomposed) into four distinct component variables (Table 4), each with their own interpretation. These variables are the mother's birth year, the mother's age at first birth, the number of years a child was born after their oldest sibling and the child's age at observation. By using these variables, instead of simply "time" of the HOME administration we are able to pinpoint which one of the separate time-related aspects is driving the change at a much finer level. For example, is the change due to ever-increasing maternal ages at first birth? Is it due to differences in children's birth cohorts? Is it due to aging of children? Or perhaps some combination is responsible. Table 4 demonstrates this decomposition using an illustrative example with two families, three children in the first and two in the second. The sum of those four variables exactly equals the year of observation, for all respondents; this arithmetic decomposition can be verified by adding the 
Table 4 Decomposition of Year of Observation into its component variables

\begin{tabular}{lllllll}
\hline Family ID & Child ID & $\begin{array}{l}\text { Mother's } \\
\text { Birth Year }\end{array}$ & $\begin{array}{l}\text { Mother's Age at } \\
\text { First Birth }\end{array}$ & $\begin{array}{l}\text { Years born after } \\
\text { the oldest sibling }\end{array}$ & $\begin{array}{l}\text { Current Age } \\
\text { Oear } \\
\text { Observed }\end{array}$ \\
\hline 1 & 1 & 1964 & 22 & 0 & 19 & 2005 \\
1 & 2 & 1964 & 22 & 2 & 17 & 2005 \\
1 & 3 & 1964 & 22 & 6 & 13 & 2005 \\
2 & 1 & 1967 & 25 & 0 & 13 & 2005 \\
2 & 2 & 1967 & 25 & 3 & 10 & 2005 \\
\hline
\end{tabular}

four values in columns 3-6 (inclusive) of Table 4 for each row. When applicable, these variables were group mean centered at the observation and child levels, with the group means reintroduced as higher level variables. Group mean centering is a common practice in multilevel modeling and is used to separate group effects (e.g., family level) from individual effects (e.g., child level; see Snijders \& Bosker, 2011). The control variables, mother's AFQT score and education as well as household income, were included in the model but were not group mean centered (as they were control variables it was only necessary that they appropriately control variance, not that their coefficients be highly interpretable and unambiguous). All analyses and data management were conducted using R (R Core Team, 2020).

\section{Results}

\section{HOME-Cognitive Stimulation Subscale}

Examining the intra-class correlation (ICC) for a three level model, the child-level ICC was less than 0.05 in each age group, but the family-level ICC was 0.62 or greater (with the exception of the youngest age group ICC $=0.25$ ). This finding suggested fitting a two level model with a family level and a combined child/observation level. For the HOME-Cognitive subscale, the overall mean (and SD), for each age group prior to imputation, from youngest to oldest was: 70.44 (14.78); 121.90 (20.01); 104.43 (23.10); 98.48 (22.29). After imputation the average mean and pooled standard deviation for each age group was: 70.32 (14.04); 121.33 (19.51); 103.46 (22.47); 97.01 (21.10). In each case there was only a slight change in mean and standard deviation after imputation, generally favoring a slightly lower mean and a reduced standard deviation.

The first, and most important, analysis examined the association between year of observation and HOME-cognitive stimulation subscale scores. This analysis was designed to evaluate whether there has been a secular change in HOMEcognitive stimulation, without concern for its causes or correlates. A model with year of observation predicting HOMEcognitive stimulation scores showed a statistically significant $(p<0.001)$, positive, effect for year of observation across all versions of the HOME at each age except for the oldest age group. The unscaled effect for each age group (in ascending age order) was: $0.84,0.70,0.39$ and 0.03 . The overall regression is shown in Fig. 1, separately by ages; a given plot is for all of the ages within a given age category.

When the control variables were added-inflation adjusted income, mother's AFQT scores and mother's education-year of observation was still positively and significantly associated with HOME-cognitive stimulation scores for the three youngest age groups (ages $0-9, p<$ 0.001 ), but not for the oldest age group. In the oldest age group the effect was actually negative. The unscaled effect for each group was $0.73,0.47,0.16$, and -0.12 . In each age group the included covariates clearly reduced the magnitude of the effect. Despite the muting effect of the control variables, after controlling for three powerful influences on children's home environment, year of observation still had an impact on HOME-cognitive stimulation scores for all but the oldest age groups (Table 5). This analysis does not describe the pattern of time-related effects at each level of analysis (year, child and family). The next analysis uses the Double Decomposition (DD) modeling strategy to account for these separate effects. The two level model fitted for the DD analysis is presented above in Equation 1.

Table 6 presents the results for the full model, with raw coefficients and their standard errors, as well as indicators of statistical significance. Pseudo-standardized effect sizes (Hoffman, 2015) can be found in Table 7, pseudostandardized effect sizes are more directly comparable than unstandardized effect sizes. Of the control variables, income was not consistently statistically significant, although it always had either no effect or a positive relation. Education's relation was similar to that of income, it never had a deleterious effect, but was not always positive. Mother's cognitive ability had a consistent positive relation. Most importantly for the present study, from among the variables partitioning year of observation, mother's age at first birth was consistently positive. For the other timerelated variables (i.e., age, child birth year and mother's birth year) the relations were occasionally significant, but the directions of the effects were inconsistent. In addition to the main results, there were additional findings we note but that were not predicted or anticipated a priori; they were not tested for statistical significance. For example, child age at observation may have a quadratic effect on the 
Fig. 1 Linear regression of cognitive stimulation subscale scores over time. Individual specific ages are plotted separately within each age group
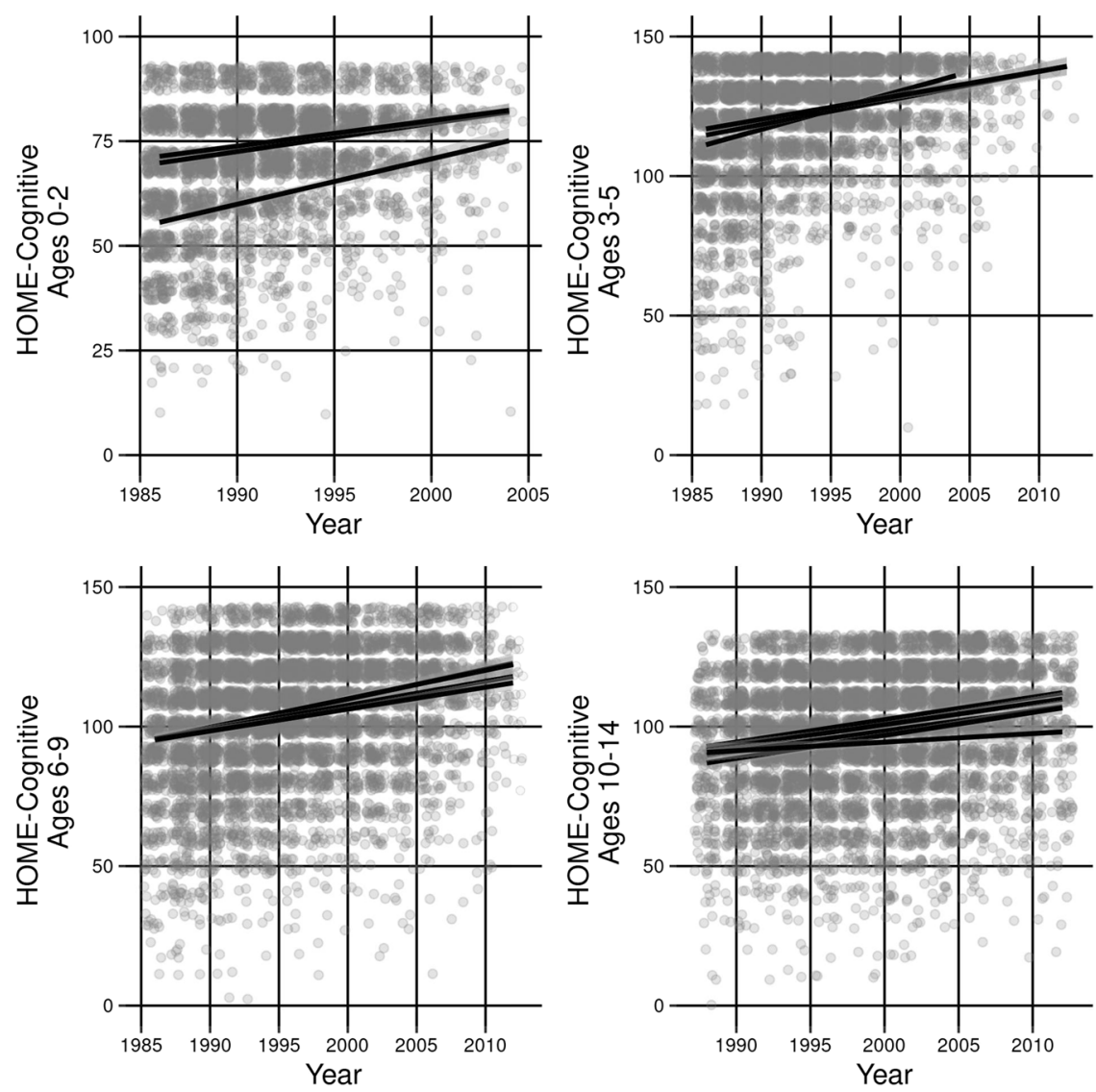

Table 5 HOME-Cognitive

Stimulation Multilevel Model B (SE); year and control variables

\begin{tabular}{lcclc}
\hline Variable & Age $0-2$ & $3-5$ & $6-9$ & $10-14$ \\
\hline Income & $0.001(0.003)$ & $0.01 * *(0.003)$ & $0.02 * * *(0.004)$ & $0.03 * * *(0.004)$ \\
Education & $0.27 *(0.12)$ & $1.25 * * *(0.16)$ & $2.08 * * *(0.16)$ & $1.49 * * *(0.15)$ \\
AFQT & $0.07 * * *(0.01)$ & $0.20^{* * *(0.01)}$ & $0.18 * * *(0.01)$ & $0.22 * * *(0.01)$ \\
Year & $0.73 * * *(0.04)$ & $0.47 * * *(0.04)$ & $0.16 * * *(0.04)$ & $-0.12 * * *(0.03)$ \\
\hline
\end{tabular}

The Armed Forces Qualifying Test score is abbreviated AFQT

$* p<0.05 ; * * p<0.01 ; * * * p<0.001$
HOME-cognitive stimulation scores, generally starting with a positive slope and becoming steadily more negative with age group. Although there may be reasonable and interpretable explanations for this, the explanations would be strictly post-hoc. In relation to the goal of our study, and the predictions that we did define a priori, these results suggest that there are more complex patterns underlying the increasing HOME-cognitive stimulation scores in relation to the four time-related predictors than a simple linear increase in scores.

\section{HOME-Emotional Support Subscale}

A similar set of analyses was conducted for the HOMEemotional support subscale. As with the cognitive stimulation subscale, evaluation of the ICC of a three level model suggested the use of a two level model, with the child level ICC never rising about 0.07 and the family level ICC between 0.31 and 0.53 . For the HOME-emotional support subscale, the overall mean (and SD), for each age group prior to imputation, from youngest to oldest was: 75.64 (12.61); 91.44 (18.90); 104.20 (18.86); 112.14 (18.89). After imputation the mean and SD for each group was: 75.59 (11.53); 91.56 (17.81); 103.95 (17.62); 111.35 (16.81). Similar to the cognitive stimulation subscale, imputed means and variances tended to be slightly lower than the complete case data. First we examined the association between year of observation and HOME-emotional support subscale scores. A multilevel model with year as the sole independent variable predicting HOME-emotional support scores showed a statistically significant $(p<0.05)$, positive, effect for year of observation across all versions of 
Table 6 HOME-Cognitive Stimulation Multilevel Model B (SE); decomposed year and control variables
Table 7 Pseudo-standardized Coefficients for full multilevel models

\begin{tabular}{|c|c|c|c|c|}
\hline Variable & Age $0-2$ & $3-5$ & $6-9$ & $10-14$ \\
\hline Income & $0.002(0.003)$ & $0.01 * *(0.003)$ & $0.02 * * *(0.004)$ & $0.03 * * *(0.004)$ \\
\hline Education & $0.17(0.12)$ & $0.95 * * *(0.16)$ & $1.68 * * *(0.17)$ & $1.05 * * *(0.15)$ \\
\hline AFQT & $0.06 * * *(0.01)$ & $0.19 * * *(0.01)$ & $0.16^{* * *}(0.01)$ & $0.19 * * *(0.01)$ \\
\hline Mother's Age at First Birth & $0.76 * * *(0.06)$ & $0.74 * * *(0.08)$ & $0.58 * * *(0.08)$ & $0.52 * * *(0.07)$ \\
\hline Mother's Birth Year & $0.53 * * *(0.10)$ & $-0.07(0.14)$ & $-0.23(0.15)$ & $-0.32 *(0.14)$ \\
\hline Family Mean Age & $5.68 * * *(0.68)$ & $0.64(0.99)$ & $-0.88(0.81)$ & $-1.80 *(0.84)$ \\
\hline Child Mean Age (Centered) & $6.87 * * *(0.51)$ & $2.13 * * *(0.56)$ & $0.31(0.34)$ & $-1.63 * * *(0.38)$ \\
\hline Child Age (Centered) & $7.35 * * *(0.19)$ & $2.02 * * *(0.20)$ & $0.08(0.13)$ & $-1.10 * * *(0.09)$ \\
\hline $\begin{array}{l}\text { Family Mean Years Born } \\
\text { After Oldest }\end{array}$ & $0.33 * * *(0.07)$ & $0.28 *(0.11)$ & $-0.21(0.14)$ & $-0.28^{*}(0.14)$ \\
\hline $\begin{array}{l}\text { Years Born After Oldest } \\
\text { (Centered) }\end{array}$ & $0.37 * * *(0.06)$ & $0.35 * * *(0.05)$ & $0.10 *(0.04)$ & $-0.07 *(0.04)$ \\
\hline
\end{tabular}

$* p<0.05 ; * * p<0.01 ; * * * p<0.001$

\begin{tabular}{|c|c|c|c|c|c|c|c|c|}
\hline \multirow[t]{2}{*}{ Variable } & \multicolumn{4}{|c|}{ Emotional Support Subscale } & \multicolumn{4}{|c|}{$\begin{array}{l}\text { Cognitive Stimulation } \\
\text { Subscale }\end{array}$} \\
\hline & $0-2$ & $3-5$ & $6-9$ & $10-14$ & $0-2$ & $3-5$ & $6-9$ & $10-14$ \\
\hline Income & 0.07 & 0.11 & 0.11 & 0.08 & 0.02 & 0.05 & 0.07 & 0.1 \\
\hline Education & -0.01 & 0.03 & 0.04 & 0.02 & 0.06 & 0.19 & 0.3 & 0.2 \\
\hline AFQT & 0.22 & 0.34 & 0.3 & 0.27 & 0.18 & 0.46 & 0.35 & 0.46 \\
\hline Mother's Age at First Birth & 0.35 & 0.53 & 0.21 & 0.02 & 0.39 & 0.34 & 0.24 & 0.23 \\
\hline Mother's Birth Year & 0.04 & 0.07 & 0.01 & -0.04 & 0.11 & -0.01 & -0.04 & -0.06 \\
\hline Family Mean Years Born After Oldest & 0.14 & 0.17 & 0 & -0.1 & 0.13 & 0.08 & -0.04 & -0.06 \\
\hline Years Born After Oldest (Centered) & 0.22 & 0.22 & 0.05 & -0.07 & 0.14 & 0.08 & 0.02 & -0.02 \\
\hline Family Mean Age & -0.05 & -0.03 & -0.01 & -0.03 & 0.16 & 0.01 & -0.02 & -0.05 \\
\hline Child Mean Age (Centered) & -0.07 & 0 & 0.02 & -0.04 & 0.29 & 0.05 & 0.01 & -0.04 \\
\hline Child Age (Centered) & -0.1 & 0.08 & 0.04 & -0.05 & 0.78 & 0.12 & 0.01 & -0.11 \\
\hline
\end{tabular}

the HOME at each age with the exception of the oldest age group (Fig. 2). The observed unscaled parameter values at each age (in ascending order of age group) were: $0.55,1.02$, 0.36 , and -0.12 . When the control variables were addedinflation adjusted income, mother's AFQT scores and mother's education-year of observation was still positively and significantly associated with HOME-emotional support scores for the three youngest age groups (ages $0-9, p<$ 0.05; Table 8), but was negative for the oldest age group. Double Decomposition models identical to those fit for the HOME-cognitive stimulation subscales were fit next.

The models produced results somewhat similar to those found for cognitive ability (Tables 7 and 9). As predicted, mother's age at first birth generally had a strong positive effect, the sole exception is for the oldest age group. As with the cognitive stimulation subscale there is generally a moderate to strong effect for mother's cognitive ability. In addition, there is a potential quadratic effect for child age at both the within-child and between-child level. Education has a surprisingly weak effect, it is not significant for any age group in any direction. Income had a generally positive effect. Lastly, the child spacing variable, years born after the oldest child, is positive at both the within- and betweenfamily level for the two youngest age groups, but negative in the oldest age group (the effect was inconsistent between levels for the 6-9 year old age group). This implies a possible interaction between the effect of child spacing and the age of the children, with larger spacing being better for younger children but worse for older.

\section{Discussion}

Our results show that the home environment of children living in the U.S., as measured by the NLSY HOME-SF, has improved over time, for all ages before adolescence between the mid-1980's and around 2015. For both the cognitive stimulation and the emotional support subscale, the longitudinal improvement is roughly equal in magnitude to the Flynn effect for intelligence, at least at younger ages 
Fig. 2 Linear regression of emotional support subscale scores over time. Individual specific ages are plotted separately within each age group
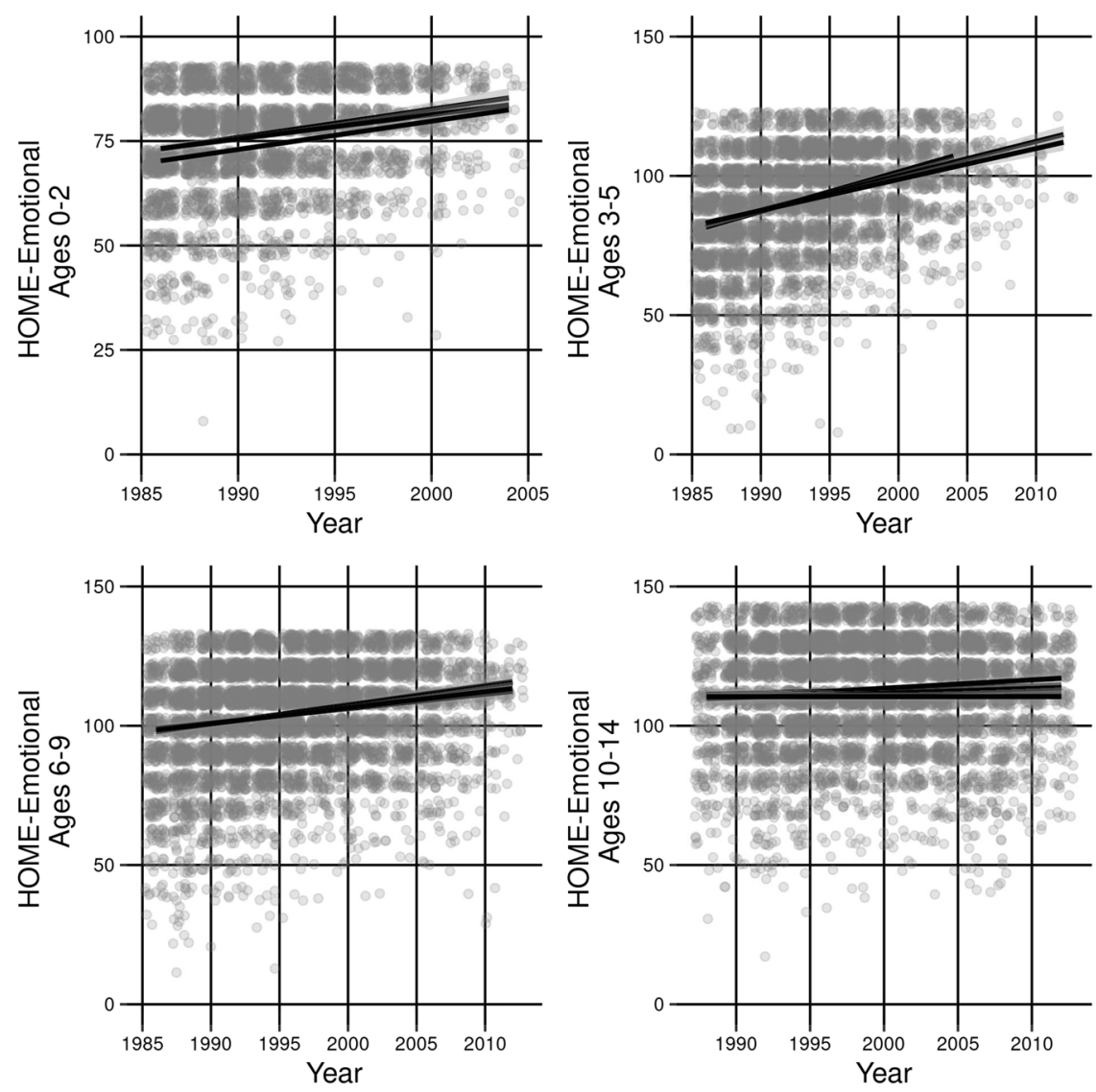

Table 8 HOME-Emotional Support Multilevel Model B (SE); year and control variables

\begin{tabular}{lclcr}
\hline Variable & Age $0-2$ & $3-5$ & $6-9$ & $10-14$ \\
\hline Income & $0.006 *(0.003)$ & $0.02 * * *(0.003)$ & $0.02 * * *(0.003)$ & $0.02 * * *(0.004)$ \\
Education & $0.24 *(0.10)$ & $0.50^{* * *}(0.14)$ & $0.42 * *(0.14)$ & $0.31 *(0.13)$ \\
AFQT & $0.08 * * *(0.01)$ & $0.15 * * *(0.01)$ & $0.14 * * *(0.01)$ & $0.12 * * *(0.01)$ \\
Year & $0.44 * * *(0.04)$ & $0.84 * * *(0.04)$ & $0.23 * *(0.03)$ & $-0.21 * * *(0.03)$ \\
\hline
\end{tabular}

$* p<0.05 ; * * p<0.01 ; * * * p<0.001$ (e.g., Pietschnig \& Voracek 2015). Our primary hypothesis, that there would be a general improvement in the home environment, and that the improvement would be similar in magnitude to the Flynn effect, were largely supported: scores have increased, and they have increased at a rate comparable to the Flynn effect (approximately 0.02 SD per year, or a raw effect size greater than at least 0.3 , Table 5). Furthermore, that increase can be attributed specifically to mother's age at first birth (or related variables, although most of the related variables are also controlled as well), similar to previous findings (e.g., Fulco et al., 2019; O'Keefe \& Rodgers, 2017). The increasing patterns are found primarily for infants and younger children. We do not propose in this paper that mother's age at first birth is the cause of this change, rather we suggest that attributes associated with mother's age at first birth (e.g., maternal traits that lead to later age at first birth) are likely candidates for the cause of this improvement. Socioeconomic variables (i.e., education and income) had varying, and relatively small, effects. The effects that were present for education and income appeared to be more prominent in the cognitive stimulation subscale rather than the emotional support subscale. Apart from the SES variables and maternal age at first birth, the only variable to have a strong and consistent effect was maternal cognitive ability as measured by the AFQT. Maternal cognitive ability and age at first birth were consistently the strongest predictors in the model, after standardizing coefficients. In only one model was an effect stronger than one of these effects (maternal education was slightly stronger than maternal age at first birth for the 6-9 year olds on the cognitive stimulation subscale, education was a weaker predictor than maternal cognitive ability). 
Table 9 HOME-Emotional Support Multilevel Model B (SE); decomposed year and control variables

\begin{tabular}{lrrrr}
\hline Variable & \multicolumn{1}{c}{ Age 0-2 } & \multicolumn{1}{c}{$3-5$} & \multicolumn{1}{c}{$6-9$} & \multicolumn{1}{c}{$10-14$} \\
\hline Income & $0.004(0.003)$ & $0.02 * * *(0.003)$ & $0.02 * * *(0.003)$ & $0.02 * * *(0.004)$ \\
Education & $-0.03(0.10)$ & $0.12(0.15)$ & $0.16(0.14)$ & $0.10(0.14)$ \\
AFQT & $0.07 * * *(0.01)$ & $0.14 * * *(0.01)$ & $0.13 * * *(0.01)$ & $0.11 * * *(0.01)$ \\
Mother's Age at First Birth & $0.64 * * *(0.05)$ & $1.16 * * *(0.07)$ & $0.48 * * *(0.06)$ & $0.04(0.07)$ \\
Mother's Birth Year & $0.19 *(0.09)$ & $0.37 * *(0.13)$ & $-0.07(0.12)$ & $-0.20(0.12)$ \\
Family Mean Age & $-1.60 * *(0.58)$ & $-1.37(0.91)$ & $-0.47(0.67)$ & $-1.20(0.80)$ \\
Child Mean Age (Centered) & $-1.31 * *(0.48)$ & $0.04(0.56)$ & $0.58(0.32)$ & $-1.36 * * *(0.40)$ \\
Child Age (Centered) & $-0.72 * * *(0.19)$ & $1.17 * * *(0.20)$ & $0.48 * * *(0.13)$ & $-0.40 * * *(0.09)$ \\
Family Mean Years Born & $0.34 * * *(0.06)$ & $0.60 * * *(0.10)$ & $0.00(0.11)$ & $-0.46 * * *(0.13)$ \\
After Oldest & & & & $-0.24 * * *(0.04)$ \\
Years Born After Oldest & $0.44 * * *(0.05)$ & $0.76 * * *(0.05)$ & $0.16 * * *(0.04)$ & \\
Centered) & & & & \\
\hline$* p<0.05 ; * p<0.01 ; * * * p<0.001$ & & & &
\end{tabular}

These predictors were generally stronger for the cognitive stimulation subscale than the emotional support subscale. These findings pair well with previous findings that maternal cognitive ability and the home environment predict subsequent childhood cognitive development (e.g., Tong et al., 2007). Previous research has suggested that the home environment may partially mediate the effect of maternal cognitive ability (e.g, Bradley et al., 1993), and additional research has shown that parental behaviors have influence on child outcomes (e.g., Bono et al., 2016). At some ages, and particularly for the cognitive stimulation subscale, these factors had sizable, independent, effects on increases in the home environment. Somewhat surprisingly, family income had a muted effect on increases in the child home environment. Previous research has indicated that socioeconomic variables have a significant influence on child cognitive outcomes, and it is somewhat surprising that we found weak relationships between similar measures of socioeconomic status and the home environment (e.g., Brooks-Gunn et al., 1996). One reason for this lack of finding may be the relatively restricted range of the HOMESF. Ceiling effects are apparent in the plots for all ages and both subscales. This restriction of range suggests that some of our estimates may have a downward bias.

Of further interest, it is worth putting our results in the context of prior research. Fulco et al. (2019), found that the HOME was related to maternal age at first birth, just as we have. In our context we have linked a longitudinal improvement in the home environment to maternal age at first birth. Given the well-known increase in maternal age at first birth over the past several decades, this finding is in line with what would be expected given previous results. What is, perhaps, less expected is that this relation does not appear to be attributable to an increased income, education or intelligence among women with a later first birth. As these variables were controlled in our models we must conclude that the effects of maternal age at first birth are not 
simply a reflection of socioeconomic differences. Furthermore, our research suggests improvements in the home environment of children that are not genetic in nature, despite evidence that much of the relationship between the home environment and child outcomes may be genetic in nature (e.g., Puglisi et al., 2017; van Bergen et al., 2017). It seems unlikely though that maternal age is anything but a general indicator of other variables. Our conclusion is that maternal age at first birth encompasses important determinants of child outcomes beyond the typical measures of SES or intelligence. During the course of our investigation, it was suggested that increased bodily autonomy for women may be a plausible explanation, as this could improve parenting self-efficacy and the readiness of the home for children. We recommend this suggestion for future research, but offer some challenges to this interpretation. First, the bulk of the NLSY childbearing occurred in the late 1980's through the early 2010's. This was a time period where the demographic transition was already completed in the U.S. and artificial methods of birth control had been in wide use for several decades. Although it remains an empirical question, large changes in bodily autonomy over the timespan studied would need to be demonstrated. Further, our own results may undermine this theory. If child spacing is accepted as an (arguable) proxy for the sort of processes being considered, our child spacing variable was not consistently statistically significant, and for older children was even negatively associated. At minimum this finding implies that the result is more nuanced, with potential benefits for younger children and potential detriments for older children. The general question "what is it about maternal age at first birth" is certainly a fruitful area for future research.

A number of effects emerged that were unrelated to our initial hypotheses. The effect of child age was inconsistent and may be quadratic in nature across the course of development. The effect of birth spacing was similarly inconsistent. These findings may be artifacts of our data or they may represent real (though subtle) effects. Of particular note is the lack of effect for the oldest age group, for both the emotional support and the cognitive stimulation subscale. This lack of effect suggested that the home environment has not significantly improved for older children. However, the effect of mother's age at first birth was still relatively strong (and significant) for children's cognitive stimulation, even in the older age group. This result suggests that other time-related trends may be occurring for the older age group that are canceling out the effect of mother's age at first birth. What these trends are and why they exist for older and not for younger children, deserves further research. Considering the generally reduced influence of parents and the family as children age would be a good starting point. Similarly, emotional support saw no benefit from maternal age at first birth for the oldest age group. Again, this finding warrants future consideration, and suggests that at older ages the influences on the home environment begin to diverge for cognitive stimulation and emotional support. These results further suggest that there are multiple causes underlying any general improvement in the home environment.

Our study was not without limitations. The design of the NLSY results in younger children being born to older mothers on average. Older mothers tend to have higher levels of cognitive ability, education and income. Although we attempted to control for these potential confounds with carefully chosen covariates, it is possible that we did not account for every possible confound. Our findings are limited to the United States during the period studied. Although the general mechanisms underlying the effects we observe may be present in other nations, this is an empirical question and should be addressed using data from those countries. Additionally, the HOME-SF is not a perfect measure of the home environment as well. It is multifaceted, and although that captures a broad picture of the home environment, it also introduces potential measurement difficulties associated with multi-factorial questionnaires. For example, two scores on the HOME-SF may be equal, but if the HOME-SF measures multiple facets of the environment equal scores could have different meanings. For our present study this was not especially concerning, as our goal was to assess broad secular changes in the home environment. Moving forward, however, and exploring what specifically has changed over time, may prove difficult with a measure such as the HOME-SF. The HOME-SF, as administered by the NLSY, used a more adhoc development process for the creation of the HOME-SF scale for the oldest age group of children. This ad-hoc development may help explain some of the differences in this age group versus the younger age groups. Furthermore, the administration process of the HOME-SF changes as children age, both the questions asked and who answers them. Answers for younger children are provided by observers and the parents. As children age they begin to provide answers for themselves. Additionally, NLSY observers were subject to change over time, and so the observers were not consistent across the entire period of the study. Finally, the reliability of the measure is relatively low for the youngest age group at approximately 0.50 (Mott, 2004), although acceptable for older age groups. However, in the current context, a lower reliability would generally lead to attenuation of effects, which suggests that our results may be an underestimate of the true effect.

In the future it will be important to determine what has caused the changes we observe. A number of state and federal policies have been implemented over the past several decades, as well as an increasing dissemination of research 
results and the potential for increased parental awareness regarding ways to improve their children's cognitive stimulation and emotional support. However, it is worth noting that the research on the impact of various policies suggests effects of large national initiatives may have small or inconsistent effects (e.g., Hamad \& Rehkopf, 2016; Jones Harden et al., 2012; Kendrick et al., 2000). Small effects on national scales can be important, but it is also important to acknowledge that our findings, and the findings of others, do not suggest that the home environment is easily manipulated by outside influence. Also of interest is the equitability of the phenomenon. It is well known that there are large racial and ethnic disparities in the U.S. on measures of SES and related variables. To the extent that these resources are important for improving the home environment we may expect differences between the home environments of different racial and ethnic groups. At minimum we would expect that the groups baselines are different, but it is also possible that the rate of change over time has favored one or more groups over others. Unfortunately, because of the design of the NLSY, any analysis of this phenomenon in the NLSY would be limited as the measures of race and ethnicity in our data are themselves limited.

Our results represent an initial foray into the exploration of changing children's home environment. The context of our study (the U.S.) is diverse and changing, both demographically and socially. It is plausible, and perhaps likely, that the changes we document will be found to vary across context. Future exploration of these changes in various socioeconomic, racial, ethnic, and geographic segments of the population is warranted. Further exploration of the impacts of more recent policies on the home environment of children (e.g., the recent stimulus payments from the Covid19 pandemic response, or work-from-home policies), may provide particularly interesting contexts for future studies.

\section{Conclusion}

On the basis of prior research and theory, we proposed that measures of the home environment may show secular change for pre-adolescent children. We further suggested that for aspects of the home environment broadly related to cognitive ability we would expect positive changes similar in direction and magnitude to the Flynn effect. For aspects of the home environment related to emotional regulation and development we were more agnostic. Although our highest-level theory predicts positive change, empirical findings related to adolescent emotional health suggested the possibility that this aspect of the home environment may be degrading. For both the cognitive stimulation and emotional support facets of the home environment, we found largely harmonious results that indicated long-term improvements in both areas, for children up to around age 10 , and these improvements were roughly similar in magnitude to the Flynn effect for younger children.

\section{Data availability}

No new data were created for this project. Data are publicly available from the Bureau of Labor Statistics from nlsinfo. org. Data are cited as Bureau of Labor statistics (BLS, 2019a; BLS, 2019b).

\section{Compliance with ethical standards}

Conflict of interest The authors declare no competing interests.

IRB Statement This work was declared exempt by [BLINDED] IRB.

Publisher's note Springer Nature remains neutral with regard to jurisdictional claims in published maps and institutional affiliations.

\section{References}

Ang, S., Rodgers, J. L., \& Wanstrom, L. (2010). The Flynn Effect within subgroups in the U.S.: Gender, race, income, education, and urbanization differences in the NLSY-Children data. Intelligence, 38, 367-384.

Bates, D., Mächler, M., Bolker, B., \& Walker, S. (2014). Fitting linear mixed-effects models using lme4. arXiv preprint arXiv:1406.5823.

van Bergen, E., van Zuijen, T., Bishop, D., \& de Jong, P. F. (2017). Why are home literacy environment and children's reading skills associated? What parental skills reveal. Reading Research Quarterly, 52(2), 147-160.

Bielecki, E. M., Haas, J. D., \& Hulanicka, B. (2012). Secular changes in the height of Polish schoolboys from 1955 to 1988. Economics \& Human Biology, 10(3), 310-317.

Blumberg, S. J., Bramlett, M. D., Kogan, M. D., Schieve, L. A., Jones, J. R., \& Lu, M. C. (2013). Changes in prevalence of parentreported autism spectrum disorder in school-aged US children: 2007 to 2011-2012. National Center for Health Statistics Reports. Number 65. National Center for Health Statistics.

Bono, E. D., Francesconi, M., Kelly, Y., \& Sacker, A. (2016). Early maternal time investment and early child outcomes. The Economic Journal, 126(596), F96-F135.

Bradley, R. H., Caldwell, B. M., Rock, S. L., Hamrick, H. M., \& Harris, P. (1988). Home observation for measurement of the environment: Development of a home inventory for use with families having children 6 to 10 years old. Contemporary Educational Psychology, 13(1), 58-71. https://doi.org/10.1016/0361476X(88)90006-9.

Bradley, R. H., Whiteside, L., Caldwell, B. M., Casey, P. H., Kelleher, K., Pope, S., \& Cross, D. (1993). Maternal IQ, the home environment, and child IQ in low birthweight, premature children. International Journal of Behavioral Development, 16(1), 61-74.

Brooks-Gunn, J., Klebanov, P. K., \& Duncan, G. J. (1996). Ethnic differences in children's intelligence test scores: Role of economic deprivation, home environment, and maternal characteristics. Child development, 67(2), 396-408.

Bureau of Labor Statistics, U.S. Department of Labor. (2019). Children of the National Longitudinal Survey of Youth 1979 cohort, 1986-2012 (rounds 1-14). Columbus, OH, The Ohio State University: Center for Human Resource Research. 
Bureau of Labor Statistics, U.S. Department of Labor. (2019). National Longitudinal Survey of Youth 1979 cohort, 1979-2012 (rounds 1-25). Columbus, OH, The Ohio State University: Center for Human Resource Research.

Bureau of Labor Statistics. (n.d.). Retention | National Longitudinal Surveys. https://www.nlsinfo.org/content/cohorts/nlsy79-children/ intro-to-the-sample/retention/page/0/1.

Buuren, S. V., \& Groothuis-Oudshoorn, K. (2010). mice: Multivariate imputation by chained equations in R. Journal of Statistical Software, 45, 1-68.

Caldwell, B. M., \& Bradley, R. H. (1984). Home observation for measurement of the environment. Little Rock: University of Arkansas at Little Rock.

Caldwell, B. M., \& Bradly, R. H. (1979) Home observation for measurement of the environment. Little Rock: University of Arkansas at Little Rock.

Collishaw, S. (2015). Annual research review: Secular trends in child and adolescent mental health. Journal of Child Psychology and Psychiatry, 56(3), 370-393.

Croen, L. A., Grether, J. K., Hoogstrate, J., \& Selvin, S. (2002). The changing prevalence of autism in California. Journal of Autism and Developmental Disorders, 32(3), 207-215. https://doi.org/ 10.1023/A:1015453830880.

Davis-Kean, P. E. (2005). The influence of parent education and family income on child achievement: The indirect role of parental expectations and the home environment. Journal of family psychology, 19(2), 294.

Demerath, E. W., Towne, B., Chumlea, W. C., Sun, S. S., Czerwinski, S. A., Remsberg, K. E., \& Siervogel, R. M. (2004). Recent decline in age at menarche: The Fels Longitudinal Study. American Journal of Human Biology, 16(4), 453-457. https://doi.org/10.1002/ajhb.20039.

Dickens, W. T., \& Flynn, J. R. (2001). Heritability estimates versus large environmental effects: The IQ paradox resolved. Psychological review, 108(2), 346.

Elardo, R., \& Bradley, R. H. (1981). The Home Observation for Measurement of the Environment (HOME) Scale: A review of research. Developmental Review, 1(2), 113-145. https://doi.org/ 10.1016/0273-2297(81)90012-5.

Flynn, J. R. (1984). The mean IQ of Americans: Massive gains 1932 to 1978. Psychological Bulletin, 95(1), 29 https://doi.org/10.1037/ 0033-2909.95.1.29.

Flynn, J. R. (1987). Massive IQ gains in 14 nations: What IQ tests really measure. Psychological Bulletin, 101(2), $171 \mathrm{https}: / /$ doi. org/10.1037/0033-2909.101.2.171.

Flynn, J. R. (2018). Reflections about intelligence over 40 years. Intelligence, 70, 73-83.

Fredriks, A. M., Van Buuren, S., Burgmeijer, R. J., Meulmeester, J. F., Beuker, R. J., Brugman, E., \& Wit, J. M. (2000). Continuing positive secular growth change in The Netherlands 1955-1997. Pediatric research, 47(3), 316-323.

Fulco, C. J., Henry, K. L., Rickard, K. M., \& Yuma, P. J. (2019). Time-varying outcomes associated with maternal age at first birth. Journal of Child and Family Studies, 1-11.

Hadd, A. R., \& Rodgers, J. L. (2016). Intelligence, income, and education as potential influences on a child's home environment: A (maternal) sibling-comparison design. Developmental Psychology, 53(7), $1286 \mathrm{https} / / /$ doi.org/10.1037/dev0000320.

Hamad, R., \& Rehkopf, D. H. (2016). Poverty and child development: a longitudinal study of the impact of the earned income tax credit. American Journal of Epidemiology, 183(9), 775-784.

Hoffman, L. (2015). Longitudinal analysis: Modeling within-person fluctuation and change. New York, NY: Routledge. https://doi. org/10.4324/9781315744094.

Hoffmann, J. P., Ellison, C. G., \& Bartkowski, J. P. (2017). Conservative Protestantism and attitudes toward corporal punishment, 1986-2014. Social Science Research, 63, 81-94.
Jones Harden, B., Chazan-Cohen, R., Raikes, H., \& Vogel, C. (2012). Early Head Start home visitation: The role of implementation in bolstering program benefits. Journal of Community Psychology, 40(4), 438-455.

Kendrick, D., Elkan, R., Hewitt, M., Dewey, M., Blair, M., Robinson, J., \& Brummell, K. (2000). Does home visiting improve parenting and the quality of the home environment? A systematic review and meta analysis. Archives of disease in childhood, 82(6), 443-451.

Loesch, D. Z., Stokes, K., \& Huggins, R. M. (2000). Secular trend in body height and weight of Australian children and adolescents. American Journal of Physical Anthropology: The Official Publication of the American Association of Physical Anthropologists, 111(4), 545-556.

Lynn, R. (2009). What has caused the Flynn effect? Secular increases in the Development Quotients of infants. Intelligence, 37(1), 16-24. https://doi.org/10.1016/j.intell.2008.07.008.

Lynn, R. (2013). Who discovered the Flynn effect? A review of early studies of the secular increase of intelligence. Intelligence, 41(6), 765-769. https://doi.org/10.1016/j.intell.2013.03.008.

McLaughlin, A. E., Campbell, F. A., Pungello, E. P., \& Skinner, M. (2007). Depressive symptoms in young adults: The influences of the early home environment and early educational child care. Child Development, 78, 746-756. https://doi.org/10.1111/j.14678624.2007.01030.x.

Mingroni, M. A. (2007). Resolving the IQ paradox: Heterosis as a cause of the Flynn effect and other trends. Psychological Review, 114(3), 806 https://doi.org/10.1037/0033-295x.114.3.806.

Mott, F. L. (2004). The utility of the HOME-SF scale for child development research in a large national longitudinal survey: The National Longitudinal Survey of Youth 1979 cohort. Parenting, 4 (2-3), 259-270.

O'Keefe, P., \& Rodgers, J. L. (2017). Double decomposition of level-1 variables in multilevel models: An analysis of the Flynn effect in the NSLY data. Multivariate Behavioral Research, 52(5), 630-647. https://doi.org/10.1080/00273171.2017.1354758.

Pietschnig, J., \& Voracek, M. (2015). One century of global IQ gains: A formal meta-analysis of the Flynn Effect. Perspectives in Psychological Science, 10, 282-306. https://doi.org/10.1177/ 1745691615577701.

Puglisi, M. L., Hulme, C., Hamilton, L. G., \& Snowling, M. J. (2017). The home literacy environment is a correlate, but perhaps not a cause, of variations in children's language and literacy development. Scientific Studies of Reading, 21(6), 498-514.

R Core Team (2020). R: A language and environment for statistical computing. R Foundation for Statistical Computing, Vienna, Austria. https://www.R-project.org/.

Ree, M. J., Mullins, C. J., Mathews, J. J., \& Massey, R. H. (1982). Armed Services Vocational Aptitude Battery: Item and factor analyses of Forms 8, 9, and 10 (Rep. No. AFHRL-TR-81-55). Brooks Air Force Base, TX: Air Force Human Resources Laboratory, Manpower and Personnel Division. (NTIS No. ADA113465). https://doi.org/10.1037/e457502004-001.

Robitzsch, A., \& Grund, S. (2020). miceadds: Some Additional Multiple Imputation Functions, Especially for 'mice'. R package version 3.10-28. https://CRAN.R-project.org/package $=$ miceadds.

Rodgers, J. L. (1998). A critique of the Flynn Effect: Massive IQ gains, methodological artifacts, or both? Intelligence, 26, 337-356. https://doi.org/10.1016/S0160-2896(99)00004-5.

Rodgers, J. L. (2015). Methodological issues associated with studying the Flynn Effect: Exploratory and confirmatory efforts in the past, present, and future. Journal of Intelligence, 3, 111-120.

Rodgers, J. L., \& Wanstrom, L. (2007). Identification of a Flynn Effect in the NLSY: Moving from the Center to the Boundaries. Intelligence., 35, 187-196. https://doi.org/10.1016/j.intell.2006. 06.002 . 
Rodgers, J. L., Rowe, D. C., \& Li, C. (1994). Beyond nature versus nurture: DF analysis of nonshared influences on problem behaviors. Developmental Psychology, 30, 374-384. https://doi.org/ 10.1037/0012-1649.30.3.374.

Sarsour, K., Sheridan, M., Jutte, D., Nuru-Jeter, A., Hinshaw, S., \& Boyce, W. T. (2011). Family socioeconomic status and child executive functions: the roles of language, home environment, and single parenthood. Journal of the International Neuropsychological Society, 17(1), 120.

Sayer, L. C., Bianchi, S. M., \& Robinson, J. P. (2004). Are parents investing less in children? Trends in mothers' and fathers' time with children. American Journal of Sociology, 110(1), 1-43.

Scarr, S., \& McCarthy, K. (1983). How people make their own environments: A theory of genotype-environment effects. Child Development, 54, 424-435.

Snijders, T. A., \& Bosker, R. J. (2011). Multilevel analysis: An introduction to basic and advanced multilevel modeling. Sage.

Strauss, R. S., \& Knight, J. (1999). Influence of the home environment on the development of obesity in children. Pediatrics, 103(6), e85-e85.
Susperreguy, M. I., Di Lonardo Burr, S., Xu, C., Douglas, H., \& LeFevre, J. A. (2020). Children's Home Numeracy Environment Predicts Growth of their Early Mathematical Skills in Kindergarten. Child Development. https://doi.org/10.1111/cdev.13353.

Tennie, C., Call, J., \& Tomasello, M. (2009). Ratcheting up the ratchet: On the evolution of cumulative culture. Philosophical Transactions of the Royal Society of London B: Biological Sciences, 364(1528), 2405-2415. https://doi.org/10.1098/rstb. 2009.0052.

Tomasello, M., Kruger, A. C., \& Ratner, H. H. (1993). Cultural learning. Behavioral and Brain Sciences, 16(3), 495-511. https:// doi.org/10.1017/S0140525X0003123X.

Tong, S., Baghurst, P., Vimpani, G., \& McMichael, A. (2007), Socioeconomic position, maternal IQ, home environment, and cognitive development. The Journal of pediatrics, 151(3), 284-288.

Turkheimer, E., Haley, A., Waldron, M., D’Onofrio, B., \& Gottesman, I. I. (2003). Socioeconomic status modifies heritability of IQ in young children. Psychological Science, 14(6), 623-628. 